\title{
Nota de l'editor: Llegir el món per escriure el futur: educació crítica per enfrontar els problemes contemporanis
}

\author{
Nota del editor: Leer el mundo para escribir el futuro: educación \\ crítica para afrontar los problemas contemporáneos \\ Editor's note: Read the world to write the future: critical education \\ to address contemporary issues
}

Jordi Castellví Mata

Universidad Internacional de la Rioja

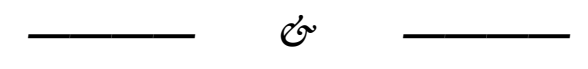

\section{NOTA DE L'EDITOR: LLEGIR EL MÓN PER ESCRIURE EL FUTUR: EDUCACIÓ CRÍTICA PER ENFRONTAR ELS PROBLEMES CONTEMPORANIS}

\begin{abstract}
Resum: La didàctica de les ciències socials i la didàctica de la llengua i la literatura estan unides per un fort vincle que es remunta a l'origen d'ambdues àrees. El desenvolupament del pensament crític i de la literacitat crítica ha estat -i segueix sentun dels elements centrals de les seves línies de recerca. Aquestes sempre han tingut en l'horitzó la formació d'una ciutadania global, reflexiva i crítica, capaç de desmantellar els relats de l'odi presents en els mitjans i en les xarxes socials, llegint més enllà de les línies i de les fonts d'informació, i analitzant textos, imatges, vídeos, $\mathrm{i}$ altres productes culturals per tal d' identificar ideologies i poder comprendre $\mathrm{i}$ enfrontar els problemes socials passats i presents, i projectar-se cap al futur.
\end{abstract}

Paraules clau: Literacitat crítica; Contrarelats de l'odi; Ciutadania global; Didàctica de les Ciències Socials 
Freire, amb la pedagogia crítica, ens va fer veure que aprendre a llegir i a escriure és un procés que va més enllà de l'aprenentatge d'unes habilitats cognitives o mecàniques. Ben al contrari, aprendre a llegir i a escriure és una pràctica de la llibertat (Freire, 2009). Freire segurament ha estat un dels pedagogs, mestres i pensadors que ha contribuït més a desdibuixar les línies que separen el que és la didàctica de la llengua i la literatura i la didàctica de les ciències socials. Aquestes línies, que de vegades semblen infranquejables, estem convençuts que no tan sols són molt fines, sinó que en molts casos són inexistents.

Autors com Paulo Freire, James Paul Gee, Allan Luke i altres ens han permès entendre la relació íntima que existeix entre paraula, pensament i acció. La lectura crítica, estudiada des de l'anàlisi crítica del discurs i la literacitat crítica son línies de recerca que serveixen de pont entre aquestes dues àrees de coneixement. Fins i tot podríem dir que, més que un simple pont, són un terreny intermedi molt fèrtil que ha permès el cultiu -la recerca- des d'ambdues àrees, amb resultats molt reeixits.

Des de la didàctica de les ciències socials el camí recorregut és llarg en aquest terreny. Fa més de vint anys que treballem en l'estudi de l'argumentació com una línia de recerca pròpia, tot i que és evident que és una línia compartida. Benejam et al. (2001) consideren que des de la perspectiva de les ciències socials "la millora de les competències psicolingüístiques de l'alumnat comporta treballar preferentment la descripció, la narració, l'explicació, la justificació i l'argumentació” (p. 57). Aquestes paraules reflecteixen el consens general del professorat de reconèixer la necessitat de processar la informació a través de la descripció i de la narració per tal de comprendre les causes $i$ les conseqüències dels problemes contemporanis. Treballs com aquest posen de manifest la importància del diàleg, la comunicació, el compartir i contrastar informació, i les argumentacions i justificacions pròpies i alienes per convèncer i per ser convençut, com a elements indispensables per poder entendre la relativitat del propi coneixement i la complexitat del coneixement social.

Altres autors també han aprofundit en l'argumentació i el debat sobre els problemes contemporanis. Gavaldà i Santisteban (2004) coincideixen en la importància d'educar per poder emetre judicis raonats a partir del treball de l'argumentació. Aquests autors van un pas mes enllà quan vinculen el desenvolupament de les habilitats argumentatives amb una de les línies de recerca centrals de la didàctica de les ciències socials: l'educació per la ciutadania crítica. Aquests autors sostenen que 
[a]rgumentar implicaria un seguit d'accions i estratègies com ara produir raons, examinar la seva rellevància i pertinència, vincular-les amb normes de caràcter universal, preveure les excepcions, trobar possibles deduccions, etc. L'argumentació comprèn, per tant, un ampli conjunt d'actituds cognitives i lingüístiques que cal organitzar. (Gavaldà i Santisteban, 2004, p. 503)

Per tant, caldria organitzar aquestes actituds cognitives i lingüístiques per tal d'aprendre i ser capaços de comunicar els significats propis de les ciències socials. En aquest sentit s'expressen Casas et al. (2005) quan defensen que "el llenguatge és l'instrument fonamental de la comunicació i que el domini de les habilitats lingüístiques permet expressar i compartir el que s'aprèn” (p.39), i ho fan per evitar caure en els dogmatismes i per poder participar en una societat democràtica tot prenent decisions i compromisos, i defensant els deures i els drets de la ciutadania. Específicament, consideren que és l'argumentació l' eina que permet desenvolupar un pensament més racional i asseguren que hem d'anar cap a la didàctica de l'argumentació per desenvolupar habilitats de pensament -des de la filosofia- i competències discursives -des de la lingüística- per construir un coneixement social crític i desenvolupar el pensament social (Canals, 2007).

Aquesta línia de recerca parteix de les actituds cognitives i lingüístiques vinculades a l'argumentació i se situa en paral·lel a l'enfocament cognitivista del desenvolupament del pensament crític que va tenir molt d'èxit durant el segle XX (Presseisen, 1986). Des d'aquesta perspectiva, el pensament crític es considera com el desenvolupament una sèrie d'habilitats de pensament. De forma similar a l'avaluació de les habilitats argumentatives (Canals, 2007), Edward Glaser (1941, citat per Presseisen, 1986) presenta un test per mesurar les capacitats per pensar de forma crítica que inclou cinc aspectes avaluables: 1) fer inferències, 2) fer assumpcions, 3) utilitzar un raonament deductiu, 4) elaborar conclusions i 5) avaluar arguments. Tant l'argumentació com la formació del pensament crític posen el focus en el desenvolupament d'una sèrie d'habilitats centrades en el raonament, en l'ús de la lògica, en el judici, etc.

L'estudi del desenvolupament del pensament crític és central en la didàctica de les ciències socials, i també s'ha abordat des d'una perspectiva cognitivista. Utilitzant el concepte de pensament social, Santisteban (2011) vincula el desenvolupament d'habilitats cognitives a la formació del pensament crític i creatiu per enfrontar els problemes contemporanis de la nostra societat. Aquest autor sosté que el pensament crític ha de basar-se en l'aprenentatge d'habilitats cognitives, però també s'ha de fonamentar en les capacitats per a valorar la informació i la seva naturalesa ideològica. A més, també inclou la importància de la intervenció social. 
En aquest punt, Santisteban (2011) fa evident un element com el pensament creatiu, que necessàriament s'ha de projectar cap a l'acció social (p. 101). El pensament crític i l'acció social son dues cares d'una mateixa moneda que sovint s'han dissociat. El salt de l'argumentació i del pensament crític cognitivista a la pedagogia crítica i a la literacitat crítica es produeix quan els mestres i els investigadors ens adonem que les habilitats de pensament no sempre suposen un impacte real en la societat i no aporten una transformació significativa de la realitat en pro de la justícia social. Tanmateix, la transició del pensament crític cognitivista a la literacitat crítica no ha estat traumàtica i no s'ha fet d'un dia per l'altre.

La literacitat crítica s'inscriu en el marc teòric de la pedagogia crítica de Paulo Freire, i s'arrela en els conceptes elaborats per aquest en els anys 70 del segle XX seguint la idea de llegir la paraula i el món per apoderar la ciutadania (Freire i Macedo, 2004). Els principis de la literacitat crítica estan emmarcats en l'aproximació de la pedagogia crítica a la formació del pensament crític (Lankshear i McLaren, 1993). Freire extreu de Marx la idea segons la qual la ideologia de la classe dominant defineix el coneixement i la ideologia escolar. Des del punt de vista de Freire, ensenyar literacitat a l'escola, és a dir, ensenyar a llegir i a escriure, s'ha fet històricament d'una manera transmisiva, des de la reproducció i la recepció passiva del coneixement. La literacitat crítica de Freire se situa en la crítica ideològica dels mitjans, la literatura, els llibres de text $i$, en definitiva, totes les produccions socials del nostre món. La idea de Freire consisteix en convertir l'alumnat en creador crític de contingut escolar i de coneixement, elaborant el seu propi currículum.

Si bé Freire obre el camí de la recerca i la pràctica de la literacitat crítica, no va ser fins als anys 90 quan va es publica per primera vegada un monogràfic on la literacitat crítica fos el tema central (Lankshear i McLaren, 1993). Aquests autors posen sobre la taula l'especial rellevància que cobraria la literacitat crítica en la societat postmoderna i ja apunten a la multiplicitat de literacitats i de relacions del llenguatge amb el poder.

A la fi del segle XX i durant les primeres dècades del segle XXI, amb l'explosió de les noves tecnologies, la literacitat crítica (com a estudi i com a enfocament educatiu) es torna encara més pertinent i sorgeixen diferents literacitats crítiques que, en tant que situades, ofereixen diferents maneres d'abordar la formació del pensament crític. En aquest sentit, la literacitat crítica dels mitjans (Alvermann i Hagood, 2000), la literacitat crítica informacional (Elmborg, 2012) o la literacitat crítica digital (Pangrazio, 2016), entre d'altres, conformen el que anomenem literacitats critiques (Mills, 2016). Les diverses literacitats crítiques ofereixen diferents 
perspectives per investigar sobre la formació del pensament crític i diferents enfocaments per desenvolupar el pensament crític a l'aula.

L'estudi de Pohl i Beaudry (2015) és una de les primeres contribucions per establir una relació explícita entre la literacitat crítica i la didàctica de les ciències socials. Aquests autors relacionen l'aprenentatge de les ciències socials amb l'alfabetització i constaten que ambdues tenen greus mancances:

Els estudiants estan aprenent a llegir i escriure, tanmateix, aquests aprenentatges poques vegades s'utilitzen per fomentar el pensament crític. Massa sovint, es veu l'alfabetització com únicament un conjunt de tècniques, funcions, coneixements i habilitats. De la mateixa manera, els estudis socials sovint també es perceben com l'aprenentatge de noms, llocs i dates. Cap d'aquestes enfocaments promou i dóna suport al pensament crític, essencial per als estudiants del segle XXI. (p. 1)

Aquests autors perceben un gran potencial en la literacitat crítica i en les ciències socials per al desenvolupament del pensament crític, no un pensament crític fonamentat en habilitats de pensament sinó un pensament crític que permeti formar a una ciutadania global crítica.

Poc després es publica la primera tesi a Espanya que vincula la didàctica de les ciències socials i la literacitat crítica (Tosar, 2017). Tosar assenyala que, fins al moment i tot i que de manera només incipient, els models de literacitat crítica sempre contemplen treballar a partir de problemes socials com a element primordial en l'ensenyament i l'aprenentatge de la història, la geografia i la ciutadania. Tosar també remarca l'important rol que hi juga l'acció social, i afirma que

[...] els estudiants poden promoure accions socials sense caure en la foscor de l'adoctrinament ideològic si se senten compromesos amb la comunitat i si han fet una verificació real de les seves experiències. En cas contrari, els nois i les noies aprendrien a llegir i escriure críticament però serien totalment incapaços de promoure accions petites i responsables per millorar la societat. Poden aprendre contingut d'estudis socials, però són lliures per marcar la diferència en aquest món. (p. 263)

Posteriorment, altres tesis també han abordat la literacitat crítica i la didàctica de la història amb l'estudi de les fonts primàries i secundàries (Godoy, 2018). Tanmateix, és a partir de la recerca portada a terme en els darrers anys liderada pel grup de recerca GREDICS, ${ }^{1}$ que la didàctica de les ciències socials a Espanya estableix relacions explícites entre la literacitat crítica, l'educació per la ciutadania

\footnotetext{
${ }^{1}$ Vegeu el projecte "Enseñar y aprender a interpretar problemas y conflictos contemporáneos: ¿Qué aportan las ciencias sociales a la formación de una ciudadanía global crítica?”, finançat pel Ministeri de Ciència i Economia del govern d'Espanya (I+D+I EDU2016-80145-P, IP: Dr. Antoni Santisteban).
} 
global i els contrarelats de l'odi, posant l'enfocament en la formació inicial del professorat i en l'educació secundària.

Aquesta recerca analitza les capacitats per a la interpretació crítica de problemes i conflictes contemporanis, des de l'ensenyament de les ciències socials, i des de la perspectiva d'una educació per a la ciutadania crítica i la justícia social. La investigació es divideix en tres grans blocs interrelacionats: a) la literacitat crítica digital enfront de problemes socials rellevants; b) els contrarelats de l'odi, les emocions i la racionalitat; i c) les persones invisibles en l'ensenyament de les ciències socials, l'humanisme radical i l'educació per a la ciutadania global. Els resultats del projecte indiquen que tant els estudiants universitaris com l'alumnat de l'educació obligatòria tenen grans dificultats per verificar la informació, diferenciar fets i opinions, descobrir silencis, interpretar arguments dels discursos de l'odi o construir contrarelats. Aquestes dificultats també sorgeixen a l'hora de comprendre la invisibilitat de persones i identitats i pensar en una ciutadania global. La investigació també ha demostrat que quan es treballa amb problemes socials i es posa a l'alumnat davant les contradiccions, falsedats i silencis de la informació, quan s'educa en la justícia social i es visibilitza aquells que són invisibles, deconstruint els arguments del discurs de l'odi, l'alumnat és capaç de descobrir la ideologia que s'amaga darrere de les línies o de les imatges, així com també de construir relats alternatius basats en els drets humans.

D'aquest projecte sorgeixen també els treballs d'Izquierdo (2019) i Castellví (2019), que estudien l'elaboració de contrarelats de l'odi i de la literacitat crítica digital. Aquestes dues línies suposen per l'àrea una aportació innovadora que encara té molt de recorregut. Izquierdo (2019) afirma que

[...] entenem com a contrarelat de l'odi [...] aquella narració que mostra una alternativa a la narració establerta. Segons De Latour et al. (2017), els relats contraris pretenen desacreditar i desconstruir les històries que serveixen de base, i també proposen alternatives orientades cap als drets humans i els valors democràtics, com ara el respecte a la diferència, la llibertat i la igualtat. Es poden fer proporcionant informació alternativa i precisa utilitzant l'humor o apel-lant a les emocions sobre els temes involucrats i explicant diferents perspectives i punts de vista. (p. 68)

La definició proposada per Izquierdo i altres treballs sobre els contrarelats de l'odi (Estellés y Castellví, 2020; Massip et al., 2020) constaten l'obertura de noves vies d'investigació des de la didàctica de les ciències socials que es recolzen en la defensa dels drets humans i dels valors democràtics enfront dels relats de l'odi. Aquests estudis també aborden alguns elements de gran importància i que estan suposant temes molt potents per a la recerca, com són les emocions o la llibertat 
d'expressió, i com aquestes s'articulen amb el desenvolupament del pensament crític.

Al seu torn, el treball de Castellví (2019) posa les bases de la literacitat crítica digital en la didàctica de les ciències socials. Sosté que la literacitat crítica digital neix com a evolució natural de la literacitat crítica tradicional, la dels mitjans (critical media lieracy) i la de les noves literacitats (new literacies). Aquest autor conceptualitza tres categories o dimensions per la comprensió, l'ensenyament i l'estudi del desenvolupament del pensament crític:

i) L'actitud d'alerta és l'estat de dubte i de reflexió davant de qualsevol tipus d'informació i davant dels problemes socials, així com la permanent revisió dels propis criteris.

ii) Els criteris estan fonamentats en els coneixements socials i en els valors democràtics i han de servir per poder elaborar argumentacions complexes.

iii) La pràctica social és la darrera dimensió del pensament crític i ha de servir per actuar i transformar la societat a través d'accions quotidianes com el de diàleg, la participació, l'apoderament i el treball per la justícia global.

La literacitat crítica digital i els contrarelats de l'odi són dues de les línies utilitzades des de la didàctica de les ciències socials per formar el pensament crític $i$, per tant, una ciutadania global crítica, que tingui esperança en la democràcia i que es projecti cap al futur. Els camins pels quals transita la didàctica de la llengua i la literatura i la didàctica de les ciències socials són comuns. L'objectiu d'ambdues àrees consisteix a formar nens i nenes, mestres, professors i professores, ciutadania, capaços de comprendre el món, de llegir-lo, d'interpretar-lo, de fer-se preguntes i d'actuar davant dels grans reptes als quals ens enfrontem com a humanitat. No estem parlant de metàfores, en els darrers anys hem viscut moments de gran transcendència i de reivindicacions col·lectives, així com grans tragèdies fruit del terrorisme global, les crisis econòmiques o la pandèmia.

En el present monogràfic constatem que l'educació crítica és i ha de ser el pilar per enfrontar tots aquests problemes contemporanis, per llegir el món i per escriure el nostre futur. Per fer-ho, comptem amb l'Amurabi Oliveira, professor de la Universitat Federal de Santa Catarina (Brasil) com a autor convidat amb l'article "Reading the world through the educational curriculum: The Social Sciences curriculum in Brazil in the context of the rise of conservatism" que ens presenta l'empremta de l'ascens del conservadorisme a Brasil en el currículum de les ciències socials i humanes. També comptem amb tres articles escrits per membres del grup de recerca GREDICS conjuntament amb professors i professores d'altres universitats espanyoles. Aquests reflecteixen el treball fet durant els últims anys des de la 
didàctica de les ciències socials per entendre com aprenem i ensenyem a llegir el món per escriure el futur. Vertebrem aquest estudi a través de tres grans temes que es corresponen amb les recerques amb alumnat d'educació secundària presentades en cada un d'aquests articles: la literacitat crítica ("Young people confronting the challenge of reading and interpreting a digital world”, Jordi Castellví, Breogán Tosar i Antoni Santisteban), la ciutadania global ("Invisibles y ciudadanía global en la formación del profesorado de educación secundaria”, Maria Ballbé, Gustavo González-Valencia i Delfín Ortega-Sánchez) i els contrarelats de l'odi ("Contrariar el odio: los relatos del odio en los medios digitales y la construcción de discursos alternativos en alumnado de educación secundaria”, Mariona Massip, Carmen Rosa García Ruiz i Neus González-Monfort). En tercer lloc, presentem una ressenya crítica escrita per Daniel Abril (Universidad de Alcalá) sobre el Handbook of Research on Citizenship and Heritage Education, que posa de relleu la importància de l'estudi del patrimoni i de la lectura del passat per ajudar a la ciutadania a comprendre el present i construir el futur. Finalment, comptem amb una entrevista als professors E Wayne Ross (University of Britsh Columbia, Canadà) i Xosé Manuel Souto (Universitat de València, Espanya) que debaten sobre la importància dels estudis socials crítics per comprendre un món globalitzat en plena pandèmia.

Esperem que el contingut d'aquest número ens permeti comprendre millor el món i els seus problemes, i ens doni algunes pistes per a transformar-lo.

\section{AGRAÏMENTS}

El editor agraeix als següents revisors i revisores per la seva contribució en la preparació del volum: Carmen Escribano (Universidad Internacional de la Rioja); Delfín Ortega-Sánchez (Universidad de Burgos); Manuel López-Martínez (Universidad de Almería); María Consuelo Díez-Bedmar (Universidad de Jaén); Marta Estellés (Universidad de Cantabria); Montserrat Yuste (Escoles Garbí).

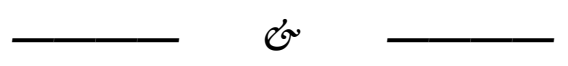




\title{
JORDI CASTELLVÍ MATA
}

Jordi Castellví és graduat en Història i Educació Primària, i doctor en Educació (2019). Els seus interessos són les metodologies d'investigació, l'educació per a la ciutadania global, el fenomen del populisme i el desenvolupament del pensament crític en contextos digitals. Castellví va comptar amb una beca de el Ministeri d'Economia i Competitivitat d'Espanya com a part de el projecte I+D+i EDU2016-80145-P de la Universitat Autònoma de Barcelona, on ha treballat com a professor d'Educació en Ciències Socials. Actualment és professor a la UNIR (Universidad Internacional de la Rioja).

jordi.castellvimata@uab.cat https://orcid.org/0000-0002-6487-5477

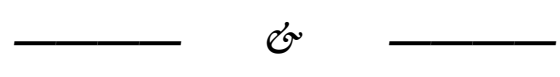

\section{NOTA DEL EDITOR: LEER EL MUNDO PARA ESCRIBIR EL FUTURO: EDUCACIÓN CRÍTICA PARA AFRONTAR LOS PROBLEMAS CONTEMPORÁNEOS}

\begin{abstract}
Resumen: La didáctica de las ciencias sociales y la didáctica de la lengua y la literatura están unidas por un fuerte vínculo que se remonta al origen de ambas áreas. El desarrollo del pensamiento crítico y de la literacidad crítica ha sido -y sigue siendo- uno de los elementos centrales de sus líneas de investigación. Estas siempre han tenido en el horizonte la formación de una ciudadanía global, reflexiva y crítica, capaz de desmantelar los relatos del odio presentes en los medios y en las redes sociales, leyendo más allá de las líneas y de las fuentes de información, y analizando textos, imágenes, vídeos, y otros productos culturales con el fin de identificar ideologías y poder comprender y enfrentar los problemas sociales pasados y presentes, y proyectarse hacia el futuro.
\end{abstract}

Palabras clave (4): Literacidad crítica; Contrarrelatos del odio; Ciudadanía global; Didáctica de las ciencias sociales.

Freire, con la pedagogía crítica, nos hizo ver que aprender a leer y escribir es un proceso que va más allá del aprendizaje de unas habilidades cognitivas o mecánicas. Al contrario, aprender a leer y escribir es una práctica de la libertad (Freire, 2009). Freire seguramente ha sido uno de los pedagogos, maestros y pensadores que ha contribuido más a desdibujar las líneas que separan lo que es la didáctica de la lengua y la literatura y la didáctica de las ciencias sociales. Estas líneas, que a veces parecen infranqueables, estamos convencidos de que no sólo son muy delgadas, sino que en muchos casos son inexistentes.

Autores como Paulo Freire, James Paul Gee, Allan Luke y otros nos han permitido entender la relación íntima que existe entre palabra, pensamiento y acción. La lectura crítica, estudiada desde el análisis crítico del discurso y la literacidad crítica son líneas de investigación que sirven de puente entre estas dos áreas de conocimiento. Incluso podríamos decir que, más que un simple puente, son un 
terreno intermedio muy fértil que ha permitido el cultivo -la investigación- desde ambas áreas, con resultados muy exitosos.

Desde la didáctica de las ciencias sociales el camino recorrido es largo en este terreno. Llevamos más de veinte años trabajando en el estudio de la argumentación como una línea de investigación propia, aunque es evidente que es una línea compartida. Benejam et al. (2001) consideran que desde la perspectiva de las ciencias sociales "la mejora de las competencias psicolingüísticas del alumnado conlleva trabajar preferentemente la descripción, la narración, la explicación, la justificación y la argumentación” (p. 57). Estas palabras reflejan el consenso general del profesorado de reconocer la necesidad de procesar la información a través de la descripción y de la narración para comprender las causas y las consecuencias de los problemas contemporáneos. Trabajos como éste ponen de manifiesto la importancia del diálogo, la comunicación, el compartir y contrastar información, y las argumentaciones y justificaciones propias y ajenas para convencer y para ser convencido, como elementos indispensables para poder entender la relatividad del propio conocimiento y la complejidad del conocimiento social.

Otros autores también han profundizado en la argumentación y el debate sobre los problemas contemporáneos. Gavaldà y Santisteban (2004) coinciden en la importancia de educar para poder emitir juicios razonados a partir del trabajo de la argumentación. Estos autores van un paso más allá cuando vinculan el desarrollo de las habilidades argumentativas con una de las líneas de investigación centrales de la didáctica de las ciencias sociales: la educación para la ciudadanía crítica. Estos autores sostienen que

\footnotetext{
[a]rgumentar implicaría una serie de acciones y estrategias tales como producir razones, examinar su relevancia y pertinencia, vincularlas con normas de carácter universal, prever las excepciones, encontrar posibles deducciones, etc. La argumentación comprende, por tanto, un amplio conjunto de actitudes cognitivas y lingüísticas que hay que organizar. (Gavaldà y Santisteban, 2004, p. 503)
}

Por tanto, habría que organizar estas actitudes cognitivas y lingüísticas para aprender y ser capaces de comunicar los significados propios de las ciencias sociales. En este sentido se expresan Casas et al. (2005) cuando defienden que "el lenguaje es el instrumento fundamental de la comunicación y que el dominio de las habilidades lingüísticas permite expresar y compartir lo que se aprende” (p.39), y lo hacen para evitar caer en los dogmatismos y para poder participar en una sociedad democrática tomando decisiones y compromisos, y defendiendo los deberes y los derechos de la ciudadanía. Específicamente, consideran que es la argumentación la herramienta que permite desarrollar un pensamiento más racional y aseguran 
que tenemos que ir hacia la didáctica de la argumentación para desarrollar habilidades de pensamiento -desde la filosofía- y competencias discursivas -desde la lingüística- para construir un conocimiento social crítico y desarrollar el pensamiento social (Canals, 2007).

Esta línea de investigación parte de las actitudes cognitivas y lingüísticas vinculadas a la argumentación y se sitúa en paralelo al enfoque cognitivista del desarrollo del pensamiento crítico que tuvo mucho éxito durante el siglo XX (Presseisen, 1986). Desde esta perspectiva, el pensamiento crítico se considera como el desarrollo de una serie de habilidades de pensamiento. De forma similar a la evaluación de las habilidades argumentativas (Canals, 2007), Edward Glaser (1941, citado por Presseisen, 1986) presenta un test para medir las capacidades para pensar de forma crítica que incluye cinco aspectos evaluables: 1) hacer inferencias, 2) hacer asunciones, 3) utilizar un razonamiento deductivo, 4) elaborar conclusiones y 5) evaluar argumentos. Tanto la argumentación como la formación del pensamiento crítico ponen el foco en el desarrollo de una serie de habilidades centradas en el razonamiento, en el uso de la lógica, en el juicio, etc.

El estudio del desarrollo del pensamiento crítico es central en la didáctica de las ciencias sociales, y también se ha abordado desde una perspectiva cognitivista. Utilizando el concepto de pensamiento social, Santisteban (2011) vincula el desarollo de habilidades cognitivas a la formación del pensamiento crítico y creativo para enfrentar los problemas contemporáneos de nuestra sociedad. Este autor sostiene que el pensamiento crítico debe basarse en el aprendizaje de habilidades cognitivas, pero también debe fundamentarse en las capacidades para valorar la información y su naturaleza ideológica. Además, también incluye la importancia de la intervención social.

En este punto, Santisteban (2011) evidencia un elemento como el pensamiento creativo, que necesariamente debe proyectarse hacia la acción social (p. 101). El pensamiento crítico y la acción social son dos caras de una misma moneda que a menudo se han disociado. El salto de la argumentación y del pensamiento crítico cognitivista a la pedagogía crítica ya la literacidad crítica se produce cuando los maestros y los investigadores nos damos cuenta de que las habilidades de pensamiento no siempre suponen un impacto real en la sociedad y no aportan una transformación significativa de la realidad en pro de la justicia social. Sin embargo, la transición del pensamiento crítico cognitivista la literacidad crítica no ha sido traumática y no se ha hecho de un día para otro. 
La literacidad crítica se inscribe en el marco teórico de la pedagogía crítica de Paulo Freire, y se arraiga en los conceptos elaborados por éste en los años 70 del siglo XX siguiendo la idea de leer la palabra y el mundo para empoderar la ciudadanía (Freire y Macedo, 2004). Los principios de la literacidad crítica están enmarcados en la aproximación de la pedagogía crítica a la formación del pensamiento crítico (Lankshear y McLaren, 1993). Freire extrae de Marx la idea según la cual la ideología de la clase dominante define el conocimiento y la ideología escolar. Desde el punto de vista de Freire, enseñar literacidad en la escuela, es decir, enseñar a leer y escribir, se ha hecho históricamente de una manera transmisiva, desde la reproducción y la recepción pasiva del conocimiento. La literacidad crítica de Freire se sitúa en la crítica ideológica de los medios, la literatura, los libros de texto y, en definitiva, todas las producciones sociales de nuestro mundo. La idea de Freire consiste en convertir al alumnado en creador crítico de contenido escolar y de conocimiento, elaborando su propio currículo.

Si bien Freire abre el camino de la investigación y de la práctica de la literacidad crítica, no fue hasta los años 90 cuando se publica por primera vez un monográfico donde la literacidad crítica es el tema central (Lankshear y McLaren, 1993). Estos autores ponen sobre la mesa la especial relevancia que cobraría la literacidad crítica en la sociedad postmoderna y ya apuntan a la multiplicidad de literacidades y de relaciones del lenguaje con el poder.

A finales del siglo XX y durante las primeras décadas del siglo XXI, con la explosión de las nuevas tecnologías, la literacidad crítica (como estudio y como enfoque educativo) se vuelve aún más pertinente y surgen diferentes literacidades críticas que, en tanto que situadas, ofrecen diferentes maneras de abordar la formación del pensamiento crítico. En este sentido, la literacidad crítica de los medios (Alvermann y Hagood, 2000), la literacidad crítica informacional (Elmborg, 2012) o la literacidad crítica digital (Pangrazio, 2016), entre otras, conforman lo que llamamos literacidades criticas (Mills, 2016). Las diversas literacitats críticas ofrecen diferentes perspectivas para investigar sobre la formación del pensamiento crítico y diferentes enfoques para desarrollar el pensamiento crítico en el aula.

El estudio de Pohl y Beaudry (2015) es una de las primeras contribuciones para establecer una relación explícita entre la literacidad crítica y la didáctica de las ciencias sociales. Estos autores relacionan el aprendizaje de las ciencias sociales con la alfabetización y constatan que ambas tienen graves carencias:

Los estudiantes están aprendiendo a leer y escribir, sin embargo, estos aprendizajes rara vez se utilizan para fomentar el pensamiento crítico. Demasiado a menudo, se ve la 
alfabetización como únicamente un conjunto de técnicas, funciones, conocimientos y habilidades. Del mismo modo, los estudios sociales a menudo también se perciben como el aprendizaje de nombres, lugares y fechas. Ninguna de estos enfoques promueve y apoya el pensamiento crítico, esencial para los estudiantes del siglo XXI. (p. 1)

Estos autores perciben un gran potencial en la literacidad crítica y en las ciencias sociales para el desarrollo del pensamiento crítico, no un pensamiento crítico fundamentado en habilidades de pensamiento sino un pensamiento crítico que permita formar una ciudadanía global crítica.

Poco después se publica la primera tesis en España que vincula la didáctica de las ciencias sociales y la literacidad crítica (Tosar, 2017). Tosar señala que, hasta el momento y aunque de manera sólo incipiente, los modelos de literacidad crítica siempre contemplan trabajar a partir de problemas sociales como elemento primordial en la enseñanza y el aprendizaje de la historia, la geografía y la ciudadanía. Tosar también remarca el importante rol que juega la acción social, y afirma que

[...] los estudiantes pueden promover acciones sociales sin caer en la oscuridad del adoctrinamiento ideológico si se sienten comprometidos con la comunidad y si han hecho una verificación real de sus experiencias. En caso contrario, los chicos y las chicas aprenderían a leer y escribir críticamente, pero serían totalmente incapaces de promover acciones pequeñas y responsables para mejorar la sociedad. Pueden aprender contenido de estudios sociales, pero son libres para marcar la diferencia en este mundo. (p. 263)

Posteriormente, otras tesis también han abordado la literacidad crítica y la didáctica de la historia con el estudio de las fuentes primarias y secundarias (Godoy, 2018). Sin embargo, es a partir de la investigación llevada a cabo en los últimos años liderada por el grupo de investigación GREDICS $^{2}$, que la didáctica de las ciencias sociales en España establece relaciones explícitas entre la literacidad crítica, la educación para la ciudadanía global y los contrarrelatos del odio, poniendo el enfoque en la formación inicial del profesorado y en la educación secundaria.

Esta investigación analiza las capacidades para la interpretación crítica de problemas y conflictos contemporáneos, desde la enseñanza de las ciencias sociales, y desde la perspectiva de una educación para la ciudadanía crítica y la justicia social. La investigación se divide en tres grandes bloques interrelacionados: a) la literacidad crítica digital frente a problemas sociales relevantes; b) los contrarrelatos del odio, las emociones y la racionalidad; y c) las personas invisibles en la enseñanza de las ciencias sociales, el humanismo radical y la educación para la ciudadanía

\footnotetext{
${ }^{2}$ Véase el proyecto "Enseñar y aprender a interpretar problemas y conflictos contemporáneos: ¿Qué aportan las ciencias sociales en la formación de una ciudadanía global crítica?", Financiado por el Ministerio de Ciencia y Economía del gobierno de España (I+D+ I EDU2016 -80145-P, IP: Dr. Antoni Santisteban).
} 
global. Los resultados del proyecto indican que tanto los estudiantes universitarios como el alumnado de la educación obligatoria tienen grandes dificultades para verificar la información, diferenciar hechos y opiniones, descubrir silencios, interpretar argumentos de los discursos del odio o construir contrarrelatos. Estas dificultades también surgen a la hora de comprender la invisibilidad de personas e identidades y pensar en una ciudadanía global. La investigación también ha demostrado que cuando se trabaja con problemas sociales y se pone al alumnado ante las contradicciones, falsedades y silencios de la información, cuando se educa en la justicia social y se visibilizan aquellos que son invisibles, deconstruyendo los argumentos del discurso del odio, el alumnado es capaz de descubrir la ideología que se esconde detrás de las líneas o de las imágenes, así como también de construir relatos alternativos basados en los derechos humanos.

De este proyecto surgen también los trabajos de Izquierdo (2019) y Castellví (2019), que estudian la elaboración de contrarrelatos del odio y de la literacidad crítica digital. Estas dos líneas suponen para el área una aportación innovadora que aún tiene mucho recorrido. Izquierdo (2019) afirma que

[...] entendemos como contrarrelato del odio [...] aquella narración que muestra una alternativa a la narración establecida. Según De Latour et al. (2017), los relatos contrarios pretenden desacreditar y deconstruir las historias que sirven de base, y también proponen alternativas orientadas hacia los derechos humanos y los valores democráticos, como el respeto a la diferencia, la libertad y la igualdad. Se pueden hacer proporcionando información alternativa y precisa utilizando el humor o apelando a las emociones sobre los temas involucrados y explicando diferentes perspectivas y puntos de vista. (p. 68)

La definición propuesta por Izquierdo y otros trabajos sobre los contrarrelatos del odio (Estellés y Castellví, 2020; Massip et al., 2020) constatan la apertura de nuevas vías de investigación desde la didáctica de las ciencias sociales que se apoyan en la defensa de los derechos humanos y los valores democráticos frente a los relatos del odio. Estos estudios también abordan algunos elementos de gran importancia y que están suponiendo temas muy potentes para la búsqueda, como son las emociones o la libertad de expresión, y como éstas se articulan con el desarrollo del pensamiento crítico.

A su vez, el trabajo de Castellví (2019) pone las bases de la literacidad crítica digital en la didáctica de las ciencias sociales. Sostiene que la literacidad crítica digital nace como evolución natural de la literacidad crítica tradicional, la de los medios (critical media lieracy) y la de las nuevas literacidades (new literacies). Este autor conceptualiza tres categorías o dimensiones para la comprensión, la enseñanza y el estudio del desarrollo del pensamiento crítico: 
i) La actitud de alerta es el estado de duda y de reflexión ante cualquier tipo de información y ante los problemas sociales, así como la permanente revisión de los propios criterios.

ii) Los criterios están fundamentados en los conocimientos sociales y en los valores democráticos y deben servir para poder elaborar argumentaciones complejos.

iii) La práctica social es la última dimensión del pensamiento crítico y debe servir para actuar y transformar la sociedad a través de acciones cotidianas como el de diálogo, la participación, el empoderamiento y el trabajo para la justicia global.

La literacidad crítica digital y los contrarrelatos del odio son dos de las líneas utilizadas desde la didáctica de las ciencias sociales para formar el pensamiento crítico y, por tanto, una ciudadanía global crítica, que tenga esperanza en la democracia y que se proyecte hacia el futuro. Los caminos por los que transita la didáctica de la lengua y la literatura y la didáctica de las ciencias sociales son comunes. El objetivo de ambas áreas consiste en formar niños y niñas, maestros, profesores, ciudadanía, capaces de comprender el mundo, de leerlo, de interpretarlo, de hacerse preguntas y de actuar ante los grandes retos a los que nos enfrentamos como humanidad. No estamos hablando de metáforas, en los últimos años hemos vivido momentos de gran trascendencia y de reivindicaciones colectivas, así como grandes tragedias fruto del terrorismo global, las crisis económicas o la pandemia.

En el presente monográfico constatamos que la educación crítica es y debe ser el pilar para enfrentar todos estos problemas contemporáneos, para leer el mundo y para escribir nuestro futuro. Para ello, contamos con Amurabi Oliveira, profesor de la Universidad Federal de Santa Catarina (Brasil) como autor invitado con el artículo "Reading the world through the educational curriculum: The Social Sciences curriculum in Brazil in the contexto of the rise of conservatism" que nos presenta la huella del ascenso del conservadurismo en Brasil en el currículo de ciencias sociales y humanas. También contamos con tres artículos escritos por miembros del grupo de investigación GREDICS juntamente con profesores de otras universidades españolas. Estos reflejan el trabajo realizado durante los últimos años desde la didáctica de las ciencias sociales para entender cómo aprendemos y enseñamos a leer el mundo para escribir el futuro. Vertebramos este estudio a través de tres grandes temas que se corresponden con las investigaciones con alumnado de educación secundaria presentadas en cada uno de estos artículos: la literacidad crítica ("Young people confronting the challenge of reading and interpreting a digital world”, Jordi Castellví, Breogán Tosar y Antoni Santisteban), la ciudadanía global ("Invisibles y ciudadanía global en la formación del profesorado de Educación Secundaria”, Maria Ballbé, Gustavo González-Valencia y Delfín Ortega-Sánchez) y los contrarrelatos del odio ("Contrariar el odio: los relatos del odio en los medios 
digitales y la construcción de discursos alternativos en alumnado de educación secundaria”, Mariona Massip, Carmen Rosa García Ruiz y Neus González-Monfort). En tercer lugar, presentamos una reseña crítica escrita por Daniel Abril (Universidad de Alcalá) sobre el Handbook of Research on Citizenship and Heritage Education, que pone de relieve la importancia del estudio del patrimonio y de la lectura del pasado para ayudar a la ciudadanía a comprender el presente y construir el futuro. Finalmente, contamos con una entrevista a los profesores E Wayne Ross (University of Britsh Columbia, Canadá) y Xosé Manuel Souto (Universidad de Valencia, España) que debaten sobre la importancia de los estudios sociales críticos para comprender un mundo globalizado en plena pandemia.

Esperamos que el contenido de este número nos permita comprender mejor el mundo y sus problemas, y nos de algunas pistas para transformarlo.

\section{AgradeCimientos}

El editor agradece a los siguientes revisores por su contribución en la preparación del volumen: Carmen Escribano (Universidad Internacional de la Rioja); Delfín Ortega-Sánchez (Universidad de Burgos); Manuel López-Martínez (Universidad de Almería); María Consuelo Díez-Bedmar (Universidad de Jaén); Marta Estellés (Universidad de Cantabria); Montserrat Yuste (Escoles Garbí).

\section{JORDI CASTELLVÍ MATA}

Jordi Castellví es graduado en Historia y Educación Primaria, y doctor en Educación (2019). Sus intereses son las metodologías de investigación, la educación para la ciudadanía global, el fenómeno del populismo y el desarrollo del pensamiento crítico en contextos digitales. Castellví contó con una beca del Ministerio de Economía y Competitividad de España como parte del proyecto I $+\mathrm{D}+\mathrm{i}$ EDU2016-80145-P de la Universitat Autònoma de Barcelona, donde ha trabajado como profesor de Educación en Ciencias Sociales. Actualmente es profesor en la UNIR (Unversidad Internacional de la Rioja).

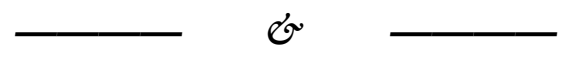




\title{
EDITOR'S NOTE: READ THE WORLD TO WRITE THE FUTURE: CRITICAL EDUCATION TO ADDRESS CONTEMPORARY ISSUES
}

\begin{abstract}
Social sciences education and language and literature education are bonded by a strong link that goes back to the origins of both areas. The development of critical thinking and critical literacy has been -and continues to be- one of the key elements of these research lines. Both have always had on the horizon a global, reflective, and critical citizenship education, capable of dismantling hate narratives present in the media and on social networks by reading beyond the lines and the sources of information, analyzing texts, images, videos, and other cultural products in order to identify ideologies and be able to understand and face past and current social issues, projecting themselves into the future.
\end{abstract}

Keywords (4): Critical Literacy; Counter-hate speech; Global citizenship; Social sciences education

Freire, with critical pedagogy, made us see that learning to read and write is a process that goes beyond learning cognitive or mechanical skills. On the contrary, learning to read and write is a practice of freedom (Freire, 2009). Freire has surely been one of the pedagogues, teachers, and thinkers who has contributed the most to blurring the lines that separate what is the didactics of language and literature and the didactics of the social sciences. We are convinced that these lines, which sometimes seem insurmountable, are not only very thin but in many cases they are non-existent.

Authors such as Paulo Freire, James Paul Gee, Allan Luke, and others have allowed us to understand the intimate relationship that exists between word, thought, and action. Critical reading, studied from critical discourse analysis and critical literacy, is a line of research that serves as a bridge between these two areas of knowledge. We could even say that more than a simple bridge, it is a very fertile intermediate ground that has allowed cultivation -research- from both areas, with very successful results.

From the didactics of the social sciences, the road traveled is long in this field. We have been working on the study of argumentation for more than twenty years as our own line of research, although it is evident that it is a shared line. Benejam et al. (2001) consider that, from a social sciences perspective, "the improvement of the psycholinguistic competences of the students entails working preferentially on description, narration, explanation, justification and argumentation" (p. 57). These words reflect the general consensus of teachers to recognize the need to process information through description and narration to understand the causes and consequences of contemporary issues. Works like this one highlight the importance of dialogue, communication, sharing and contrasting information, and the arguments and justifications of one's own and of others to convince and 
be convinced as essential elements to understand the relativity of one's own knowledge and the complexity of social knowledge.

Other authors have also delved into the argumentation and debate on contemporary issues. Gavaldà and Santisteban (2004) agree on the importance of educating in order to make reasoned judgments based on the work of argumentation. These authors go a step further when they link the development of argumentative skills with one of the central lines of research in social science didactics: critical citizenship education. These authors argue that

[a]rgumentation would imply a series of actions and strategies such as producing reasons, examining their relevance and pertinence, linking them with universal norms, anticipating exceptions, finding possible deductions, etc. The argumentation comprises, therefore, a wide set of cognitive and linguistic attitudes that must be organized. (Gavaldà \& Santisteban, 2004, p. 503)

Therefore, these cognitive and linguistic attitudes should be organized to learn and be able to communicate the meanings of the social sciences. In this sense, Casas et al. (2005) defend that "language is the fundamental instrument of communication and that the mastery of linguistic skills allows to express and share what is learned" (p.39), and they do so to avoid falling into dogmatism and to be able to participate in a democratic society by making decisions and commitments and defending the duties and rights of citizens. Specifically, they consider that argumentation is the tool that allows us to develop more rational thought and they assure that we have to go towards the didactics of argumentation to develop thinking skills -from philosophy- and discursive skills -from linguistics- to build critical social knowledge and develop social thinking (Canals, 2007).

This line of research starts from the cognitive and linguistic attitudes linked to argumentation and is situated in parallel to the cognitivist approach to the development of critical thinking that was very successful during the 20th century (Presseisen, 1986). From this perspective, critical thinking is seen as the development of a series of thinking skills. Similar to the evaluation of argumentative skills (Canals, 2007), Edward Glaser (1941, cited by Presseisen, 1986) presents a test to measure the abilities to think critically that includes five assessable aspects: 1 ) making inferences, 2) making assumptions, 3) use deductive reasoning, 4) draw conclusions, and 5) evaluate arguments. Both the argumentation and the formation of critical thinking focus on the development of a series of skills focused on reasoning, the use of logic, judgment, etc. 
The study of the development of critical thinking is central in the didactics of the social sciences and has also been approached from a cognitivist perspective. Using the concept of social thinking, Santisteban (2011) links the development of cognitive skills to the formation of critical and creative thinking to face contemporary issues in our society. This author argues that critical thinking should be based on learning cognitive skills, but it should also be based on the abilities to value information and its ideological nature. In addition, it also includes the importance of social intervention.

At this point, Santisteban (2011) includes an element such as creative thinking, which must necessarily be projected towards social action (p. 101). Critical thinking and social action are two sides of the same coin that have often been dissociated. The leap from argumentation and cognitivist critical thinking to critical pedagogy and critical literacy occurs when teachers and researchers realize that thinking skills do not always have a real impact on society and do not bring a significant transformation of reality in favor of social justice. However, the transition from cognitivist critical thinking to critical literacy has not been traumatic and it has not happened overnight.

Critical literacy is part of the theoretical framework of Paulo Freire's critical pedagogy and is rooted in the concepts elaborated by him in the 70s of the 20th century, following the idea of reading the word and the world to empower citizens (Freire \& Macedo, 2004). The principles of critical literacy are framed in the approach of critical pedagogy to critical thinking education (Lankshear \& McLaren, 1993). Freire draws from Marx the idea according to which the ideology of the ruling class defines knowledge and school ideology. From Freire's point of view, teaching literacy in school, that is, teaching to read and write, has historically been done in a transmissive way, through the reproduction and passive reception of knowledge. Freire's critical literacy is situated in the ideological critique of the media, literature, textbooks, and, ultimately, all social productions of our world. Freire's idea is to turn students into critical creators of school content and knowledge, developing their own curriculum.

Although Freire paves the way for the research and practice of critical literacy, it was not until the 1990s that critical literacy was the special issue of a handbook (Lankshear \& McLaren, 1993). These authors show the special relevance that critical literacy would acquire in postmodern society and pinpoint the multiplicity of literacies and the relationships between language and power. 
At the end of the 20th century and during the first decades of the 21 st century, with the explosion of new technologies, critical literacy (as a study and as an educational approach) becomes even more pertinent and different critical literacies emerge which, as situated, they offer different ways of approaching critical thinking education. In this sense, critical media literacy (Alvermann \& Hagood, 2000), critical informational literacy (Elmborg, 2012), and critical digital literacy (Pangrazio, 2016), among others, become what we call the critical literacies (Mills, 2016). The various critical literacies offer different perspectives for research on critical thinking education and different approaches to developing critical thinking in the classroom.

The study by Pohl and Beaudry (2015) is one of the first contributions to establish an explicit relationship between critical literacy and social science education. These authors relate the learning of the social sciences with literacy and confirm that both have serious deficiencies:

Students are learning literacy; however, literacy is seldom used to foster critical thinking. Too often, literacy is viewed narrowly as consisting solely of a set of technical and functional knowledge and skills. Similarly, social studies are often also perceived as consisting primarily of names, places, and dates. Neither of these approaches promote and supports the critical thinking that is essential for learners in the twenty-first century. (p. 1)

These authors perceive great potential in critical literacy and the social sciences for the development of critical thinking. A type of critical thinking which is not based on thinking skills but critical thinking to critical global citizenship education.

Shortly after, the first thesis was published in Spain that links the didactics of the social sciences and critical literacy (Tosar, 2017). Tosar points out that, up to now and although only incipient, critical literacy models always contemplate working with social issues as a primary element in the teaching and learning of history, geography, and citizenship. Tosar also highlights the important role that social action plays and states that

[...] students can promote social actions without falling into the darkness of ideological indoctrination if they feel committed to the community, and if they have made a real verification of their experience. Otherwise, boys and girls would learn how to read and write critically but they would, be totally unable to promote responsible, small, actions to improve the society. They can learn social studies content, but they are free to make a difference in this world. (p. 263)

Subsequently, others have also addressed the relation between critical literacy and didactics of history with the study of primary and secondary sources (Godoy, 2018). However, it is from the research carried out in recent years led by the 
GREDICS $^{3}$ research group, that the didactics of the social sciences in Spain establishes explicit relationships between critical literacy, education for global citizenship, and counter-hate speech narratives, focusing on initial teacher training and in secondary education.

This research analyzes the capacity of social sciences education and of education for critical citizenship and social justice to promote a critical interpretation of contemporary issues and conflicts. The research is divided into three large interrelated blocks: a) critical digital literacy to challenge social issues; b) counter-hate speech narratives, emotions, and rationality; and c) invisible people in social sciences education, radical humanism, and education for global citizenship. The results of the project indicate that both university students and secondary school students have great difficulties in verifying information, differentiating facts and opinions, discovering silences, interpreting hate speech arguments, or constructing counter-narratives. These difficulties also arise when it comes to understanding the invisibility of people and identities and thinking about a global citizenship. Research has also shown that when working with social issues and confronting students with contradictions, falsehoods, and silences of information, when educating themselves in social justice and making those who are invisible visible, deconstructing the arguments of hate speech, the students are able to discover the ideology beyond the lines and the images, as well as to construct alternative narratives based on human rights.

The works of Izquierdo (2019) and Castellví (2019) are also resulting from this project. They research on the education for counter-hate speech and critical digital literacy, respectively. These two research lines represent an innovative contribution to the area that still has a long way to go. Izquierdo (2019) states that

[...] we understand as a counter-hate speech narrative [...] that narrative that shows an alternative to the established discourse. According to De Latour et al. (2017), the contrary stories seek to discredit and deconstruct the stories that serve as the basis, and also propose alternatives oriented towards human rights and democratic values, such as respect for difference, freedom and equality. They can be done by providing alternative and accurate information using humor or by appealing to emotions on the issues involved and explaining different perspectives and points of view. (p. 68)

The definition proposed by Izquierdo and other works on counter-hate speech (Estellés \& Castellví, 2020; Massip et al., 2020) confirms the opening of

\footnotetext{
${ }^{3}$ See the research project "Enseñar y aprender a interpretar problemas y conflictos contemporáneos: ¿Qué aportan las ciencias sociales en la formación de una ciudadanía global crítica?”, Funded by the Spanish Ministry of Science and Economy (R\&D EDU2016 - 80145-P, PI: Dr. Antoni Santisteban).
} 
new avenues of research from the didactics of the social sciences that are based on the defense of human rights and democratic values to confront hate speech. These studies also address some elements of great importance that are becoming very powerful research topics, such as emotions or freedom of expression, and how these are articulated with the development of critical thinking.

In turn, Castellví's (2019) work establishes the foundations for critical digital literacy in the didactics of social sciences. He argues that critical digital literacy was born as a natural evolution of traditional critical literacy, that of the media and that of new literacies. This author conceptualizes three categories or dimensions for understanding, teaching, and studying the development of critical thinking:

i) The alert attitude is the state of doubt and reflection in front of any type of information and in front of social issues, as well as the permanent review of one's own criteria.

ii) The criteria are based on social knowledge and democratic values and should be used to build complex arguments.

iii) Social practice is the last dimension of critical thinking and must serve to act and transform society through daily actions such as dialogue, participation, empowerment, and work for global justice.

Critical digital literacy and counter-hate speech are two of the research lines used from the didactics of the social sciences to critical thinking education and, therefore, a critical global citizenship education, which has hope in democracy and is projected into the future. The paths through which the didactics of language and literature and the didactics of the social sciences travel are shared. The objective of both areas is to educate boys and girls, primary and secondary teachers, citizenship, capable of understanding the world, reading it, interpreting it, asking questions and acting in front of the great challenges that we face as humanity. We are not talking about metaphors, in recent years we have experienced moments of great significance and collective claims, as well as great tragedies resulting from global terrorism, economic crises, or the pandemic.

In this special issue, we confirm that critical education is and must be the pillar to face all these contemporary issues, to read the world, and to write our future. For this, we have Amurabi Oliveira, professor at the Federal University of Santa Catarina (Brazil) as a guest author with the article "Reading the world through the educational curriculum: The Social Sciences curriculum in Brazil in the context of the rise of conservatism" that presents us with the trace of the rise of conservatism in Brazil in the social and human sciences curriculum. We also have 
three articles written by members of the GREDICS research group together with professors from other Spanish universities. These reflect the work carried out in recent years from the didactics of the social sciences to understand how we learn and teach how to read the world to write the future. We structure this study through three main themes that correspond to the research with secondary school students presented in each of these articles: critical literacy ("Young people confronting the challenge of reading and interpreting a digital world", Jordi Castellví, Breogán Tosar and Antoni Santisteban ), global citizenship ("Invisibles y ciudadanía global en la formación del profesorado de educación secundaria ”, Maria Ballbé, Gustavo González-Valencia and Delfín Ortega-Sánchez) and counter-hate speech ("Contrariar el odio: los relatos del odio en los medios digitales y la construcción de discursos alternativos en alumnado de educación secundaria”, Mariona Massip, Carmen Rosa García Ruiz and Neus González-Monfort). Third, we present a critical review written by Daniel Abril (University of Alcalá, Spain) on the Handbook of Research on Citizenship and Heritage Education, which highlights the importance of studying heritage and reading the past to help citizens to understand the present and build the future. Finally, we have an interview with professors E Wayne Ross (University of British Columbia, Canada) and Xosé Manuel Souto (University of Valencia, Spain), who discuss the importance of critical social studies to understand a globalized world in the midst of a pandemic.

We hope that this issue will allow us to better understand the world and its issues and give us some clues to transform it.

\section{ACKNOWLEDGEMENTS}

The editor would like to thank the following reviewers for their contribution to the preparation of the volume: Carmen Escribano (International University of La Rioja); Delfín Ortega-Sánchez (University of Burgos); Manuel López-Martínez (University of Almería); María Consuelo Díez-Bedmar (University of Jaén); Marta Estellés (University of Cantabria); Montserrat Yuste (Escoles Garbí).

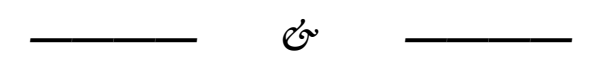

\section{REFERENCES}

Alvermann, D., \& Hagood, M. (2000). Critical media literacy: Research, theory, and practice in "new times". The Journal of Educational Research, 93(3), 193-205. http://doi.org/10.1080/00220670009598707

Benejam, P., Cases, M., Llobet, C., \& Oller, M. (2001). La justificación y argumentación de la enseñanza de las ciencias sociales. Iber, 28, 57-68. 
Canals, R. (2007). La argumentación en el aprendizaje del conocimiento social. Enseñanza de las ciencias sociales, 6, 49-60.

Casas, M., Bosch, D., \& González, N. (2005). Las competencias comunicativas en la formación democrática de los jóvenes: describir, explicar, justificar, interpretar y argumentar. Enseñanza de las ciencias sociales, 4, 39-52.

Castellví, J. (2019). Critical digital literacy in social studies. Case studies in elementary school. [Tesi doctoral]. Universitat Autònoma de Barcelona.

Elmborg, J. (2012). Critical information literacy: Definitions and challenges. A C. Wetzel \& C. Bruch (Eds.), Transforming information literacy programs: Intersecting frontiers of self, library culture, and campus community (pp. 75-95). Association of College \& Research Libraries.

Estellés, M., \& Castellví, J. (2020). The educational implications of populism, emotions and digital hate speech: A dialogue with scholars from Canada, Chile, Spain, the UK, and the US. Sustainability, 12(15), 6034. https://doi.org/10.3390/su12156034

Freire, P. (2009). La Educación como práctica de la libertad. Siglo XXI.

Freire, P., \& Macedo, D. (2004). Literacy: Reading the word and the world. Routledge.

Gavaldà, A., \& Santisteban, A. (2004). La formación de la ciudadanía. Capacidades para la argumentación. A M. I. Vera \& D. Pérez (Eds.). Formación de la ciudadanía: Las TICs y los nuevos problemas (pp. 529-539). Universidad de Alicante/Asociación Universitaria del Profesorado de Didáctica de las Ciencias Sociales.

Godoy, F. (2018). Interpretación de fuentes históricas y desarrollo de la literacidad crítica en estudiantes chilenos de educación secundaria. [Tesi doctoral]. Universitat Autònoma de Barcelona.

Izquierdo, A. (2019). Contrarelats de l'odi a l'ensenyament i aprenentatge de les ciències socials. Una recerca interpretativa i crítica a l'educació secundària. [Tesi doctoral]. Universitat Autònoma de Barcelona.

Lankshear, C., \& McLaren, P. (1993). Critical literacy. Politics, praxis, and the postmodern. State University of New York Press.

Massip, M., Anguera, C., \& Llusà, J. (2020). Deconstruir los relatos del odio en el aula. Íber, 99, 24-31.

Mills, K. A. (2016). Literacy theories for the digital age. Social, critical, multimodal, spatial, material and sensory lenses. Multilingual Matters.

Pangrazio, L. (2016). Reconceptualising critical digital literacy. Discourse: Studies in the Cultural Politics of Education, 37(2), 163-174. https://doi.org/10.1080/01596306.2014.942836

Pohl, B., \& Beaudry, C. (2015). Critical literacy in the social studies classroom: a case study for the 21st century. Journal of Family Strengths, 15(2), 1-13.

Presseisen, B. Z. (1986). Critical thinking and thinking skills: State of the art definitions and practice in public schools. Research for Better Schools, Inc.

Santisteban, A. (2011). La formación del pensamiento social y el desarrollo de las capacidades para pensar la sociedad. A A. Santisteban, \& J. Pagès (Eds.), Didáctica del conocimiento del medio social y cultural en la educación primaria: ciencias sociales para comprender, pensar y actuar (pp.85-103). Síntesis.

Tosar, B. (2017). Llegir la paraula i el món: literacitat crítica en els estudis socials a l'educació primària. [Tesi doctoral]. Universitat Autònoma de Barcelona. 


\section{JORDI CASTELLVÍ MATA}

Jordi Castellví has a degree in History and Primary Education and a PhD. in Education (2019). His interests are research methodologies, global citizenship education, the phenomenon of populism, and the development of critical thinking in digital contexts. Castellví had a scholarship from the Spanish Ministry of Economy and Competitiveness as part of the R\&D EDU2016-80145-P project at the Universitat Autònoma de Barcelona, where he has worked as a research fellow of Social Studies Education. He is currently a lecturer at the UNIR (Universidad Internacional de la Rioja).

jordi.castellvimata@uab.cat https://orcid.org/0000-0002-6487-5477

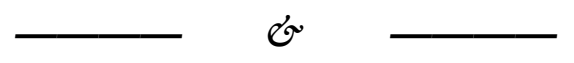

Castellví Mata, J. (2021). Nota de l'editor: Llegir el món per escriure el futur: educació crítica per enfrontar els problemes contemporanis. Bellaterra Journal of Teaching \& Learning Language \& Literature, 14(2), e982. https://doi.org/10.5565/rev/jtl3.982 